\title{
Improving early detection of developmental dysplasia of the hip through general practitioner assessment and surveillance
}

Nicole Williams

This article is the third in a series on paediatric health. Articles in this series aim to provide information about diagnosis and management of presentations in infants, toddlers and pre-schoolers in general practice.

\section{Background}

Cases of late diagnosed developmental dysplasia of the hip (DDH) have increased in Australia in recent years despite an ongoing clinical screening program and increasing use of selective ultrasonography screening.

\section{Objective \\ The aim of this paper is to discuss an evidence-based approach that involves prevention and early detection of DDH using assessment and surveillance that is suitable for general practitioners (GPs) who see young children in their practices.}

\section{Discussion}

Repeated, carefully performed clinical examinations of the paediatric hip from newborn to walking age remain the best method for early detection of DDH. GPs are ideally placed to conduct regular assessments to maximise detection of dislocated hips in early infancy and lower the age at detection for children who develop progressive signs of DDH outside the newborn period.
HIPPOCRATES promoted early treatment of infantile hip dislocations almost 2400 years ago. ${ }^{1}$ In Australia, a newborn congenital dislocation of the hip (CDH) screening program was implemented in the 1960s. However, late detection of hip dislocations, including at walking age, have increased in Australia since 2003..$^{2-4}$

$\mathrm{CDH}$ was renamed developmental dysplasia of the hip (DDH) ${ }^{5}$ and describes a range of paediatric hip conditions including instability, acetabular dysplasia, subluxation and dislocation. There is no standard definition and no consensus regarding which clinical examination and imaging findings require treatment to permit normal hip development. ${ }^{6,7}$ In South Australia, where DDH is notifiable to the South Australian Birth Defects Register, the incidence is around seven per 1000 live births. ${ }^{4}$ For children born in 2003-2009, $11.5 \%$ of cases were a late diagnosis after three months of age. ${ }^{4}$

Current DDH screening practices are criticised for failure to adhere to the general principles of health screening, ${ }^{8,9}$ and there is variability in Australian neonatal hip screening guidelines. ${ }^{10-16}$

This paper presents an evidence-based approach to DDH prevention and early detection using assessment and surveillance, suitable for general practitioners (GPs).

\section{Risk factors and prevention of DDH}

DDH results from a combination of environmental and genetic factors. ${ }^{17}$ Associated gene defects occur in the transforming growth factor beta superfamily, necessary for normal bone and joint development. ${ }^{18}$
The highest DDH risk is in female breech babies (absolute risk 120/1000), followed by females with a family history (44/1000), then male breech babies (26/1000). ${ }^{17}$ The estimated risk for males with a family history $(9.4 / 1000)$ is lower than females in general (19/1000). ${ }^{17}$ There is ongoing debate as to whether foot abnormalities and oligohydramnios are truly risk factors. ${ }^{8}$

Hip development can be affected postnatally. Tight swaddling of lower limbs in extension and adduction is associated with DDH in epidemiological, anatomical and animal studies. ${ }^{19-21} \mathrm{High}$ rates of swaddling are seen in patients with DDH diagnosed after three months of age. ${ }^{22,23}$ Conversely, habitually carrying babies with hips flexed and abducted is associated with low DDH incidence. ${ }^{20}$ Optimal babywearing has been proposed as a public health initiative to minimise DDH. ${ }^{20}$ The International Hip Dysplasia Institute maintains a list of 'hip-healthy' early childcare products. ${ }^{21}$ Reduced prone lying for babies to minimise sudden infant death syndrome and use of disposable rather than bulky cloth nappies have been postulated to contribute to late $\mathrm{DDH}$ cases, ${ }^{24}$ but this is speculative.

\section{Rationale for early detection}

Early detection for any condition seeks to identify and treat individuals who have developed pathology but not yet sought medical attention. ${ }^{9}$ DDH treatment harnesses the ossification potential of the hip to achieve a reduced, stable and mature hip, while attempting to avoid treatment complications including growth disturbance and avascular necrosis. ${ }^{25,26}$ 
Hip maturation potential is greatest during the first six weeks of life and reaches a plateau by the end of the third month. ${ }^{25}$ Brace treatment has a fourfold higher risk of failure when commenced after seven weeks of age. ${ }^{26}$ If the femoral head fails to consistently contact the acetabulum, progressive secondary changes occur, including adductor muscle shortening and mismatch in the morphology of the femoral head and acetabulum. ${ }^{17,27}$ With increasing treatment age, invasive procedures become more likely, including extensive soft tissue releases and osteotomies..$^{2,3}$ Treatment failures and complications are more common. ${ }^{4,22}$

As well as maximising the chance of successful treatment with an abduction splint or Pavlik harness, early DDH detection lowers the age at diagnosis for children who develop progressive signs outside the newborn period. The majority of children diagnosed after three months of age self-present when a limp or leg length discrepancy ${ }^{2-4}$ is noticed at walking age. However, abnormal examination findings may be detected earlier when actively sought. ${ }^{28,29}$

\section{Optimal assessment of DDH and the role of the GP}

A holistic approach to DDH assessment acknowledges that a combination of risk factor assessment, clinical examination and imaging studies may permit early detection of DDH. ${ }^{25,30}$

DDH risk factors signal a need to maintain a high index of suspicion for hip pathology. Breech babies with DDH are rarely diagnosed after three months of age, even without ultrasonography screening, presumably because awareness of the increased risk leads to careful examination by skilled examiners. ${ }^{4,28,29}$ Some Australian guidelines recommend referral of babies with risk factors, including breech birth, family history, torticollis and foot deformities, to specialist hip clinics for assessment and/or recommend ultrasonography screening. ${ }^{10-16}$ GPs who see young children should be aware of local guidelines. In Western Australia in 2010, six of 17 children with late-diagnosed $\mathrm{DDH}$ were not referred to the paediatric orthopaedic service in the neonatal period, despite meeting referral criteria. ${ }^{3}$

Clinical examination remains the most important component of assessment. Rates of late-diagnosed DDH in South Australia remained very low for decades (2.1\% of DDH cases), with an established program of hip clinical examination at birth, on each day during the hospital stay, and at well-baby clinics up to 12 months of age, with ultrasonography reserved only for equivocal clinical examination. ${ }^{29}$ Risk factors for late DDH include early hospital discharge and rural birth, ${ }^{4,28,29}$ possibly reflecting inadequate opportunity for the careful examination of a settled baby by a suitably experienced examiner. ${ }^{4}$ There was no documentation of any neonatal hip examination in $47 \%$ of Western Australian children diagnosed with DDH after six months of age in $2010 .{ }^{3}$ The GP may be the first practitioner to make an adequate assessment, even if they are not considered the usual clinician responsible for newborn hip examination. ${ }^{31}$ Assessment of the abduction range in conjunction with a gentle Ortolani manoeuvre (Figure 1) is a safe practice to detect a dislocated hip in a child who may have been unsettled at previous examination attempts. Repeated forceful examinations should be avoided. ${ }^{7,17}$ Low rates of late-diagnosis DDH are seen in babies examined on each day of postnatal hospital admission, ${ }^{29}$ supporting the utility of repeated early examinations. Clinical examination

Ortolani's test - is positive if hip is lying in a dislocated position and is manually reducible.

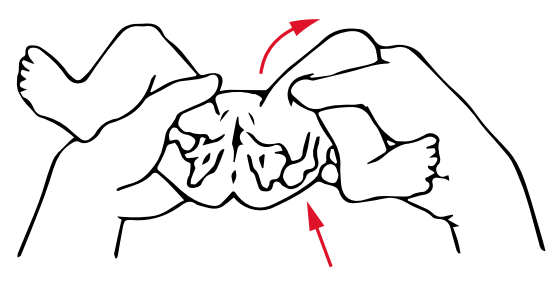

Figure 1. Ortolani manoeuvre: in a settled baby on a firm surface, the flexed and adducted hip is abducted while applying gentle traction and pressure on the greater trochanter. A dislocated hip relocates with a 'clunk'. findings should be documented and communicated specifically, ${ }^{7}$ describing the hip as dislocated, dislocatable, subluxatable or stable and documenting the abduction range.

Clinical examination in babies up to eight weeks of age is with carefully performed Ortolani (Figure 1) and Barlow (Figure 2) tests in a settled baby. ${ }^{10,17,32,33}$ An Ortolani-positive hip is dislocated at rest but reducible and requires prompt referral for treatment. Early referral of a Barlow-positive hip (resting in joint but dislocatable) minimises treatment delays for a hip that fails to stabilise. ${ }^{17}$ Although uncommon in the young child, a hip may be dislocated at rest and irreducible (negative for both Ortolani and Barlow tests). Inability to abduct both hips in a young baby so that the lateral surface of the knees contacts the examination surface may suggest an irreducible dislocation. ${ }^{15}$ An urgent ultrasound (not delaying to six weeks of age) can confirm the femoral head position relative to the acetabulum if this is suspected.

Unilateral decreased hip abduction in babies older than eight weeks suggests a dislocated or subluxated hip with $78.3 \%$ sensitivity. ${ }^{8}$ Bilateral reduced abduction is more difficult to assess and is a less specific sign. ${ }^{8}$ Ortolani and Barlow signs are often stated to be unreliable outside the newborn period but still occur. ${ }^{17}$ In a prospective study, $44 \%$ of hips dislocated at rest in children aged 3-18 months

Barlow's test - is positive if the hip can be manually dislocated.

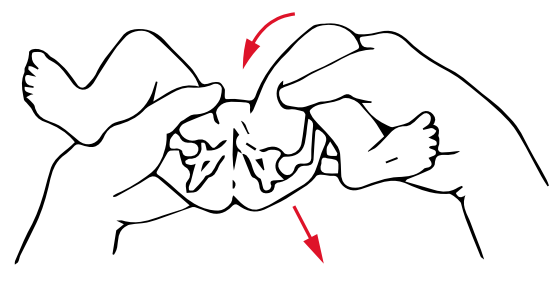

Figure 2. Barlow manoeuvre: the hip is adducted and pressure relieved from the greater trochanter. A gentle posterior force will cause a dislocatable hip to palpably slip out of the posterior acetabulum. 
were clinically reducible. ${ }^{22}$ Other signs of femoral head malposition include a positive Galeazzi test (relative shortness of the femur with the hips and knees flexed) and leg length discrepancy. ${ }^{17}$ Asymmetrical thigh or gluteal skin folds (best assessed with the child prone) ${ }^{17}$ are included in many DDH screening guidelines $^{8,10,11,13,14,16}$ but are only associated with DDH in the presence of other findings such as leg length discrepancy or decreased abduction. ${ }^{8} \mathrm{~A}$ walking-age child with unilateral $\mathrm{DDH}$ can present with leg length discrepancy, unilateral toe walking (compensatory for leg length inequality) or a limp. A child with bilateral dislocations presents with a waddling gait and hyperlordosis. ${ }^{11}$

The use of ultrasonography in DDH assessment is subject to ongoing debate. ${ }^{6}$ A 2013 Cochrane review concluded that universal ultrasonography of all newborn hips increased treatment rates without differences in late diagnosis or surgery, ${ }^{6}$ contrasting with European studies showing reductions in late detection and surgery compared with the era before ultrasonography screening. ${ }^{34,35}$ A possible explanation lies in rigorous quality control. In Germany, ultrasonography licences are withdrawn from practitioners who fail to meet centrally monitored standards. ${ }^{36}$ A range of ultrasonography techniques to identify DDH are used in Australia, sometimes in combination, including static measures of femoral head coverage and acetabular morphology $y^{25,37}$ as well as assessments of stability. ${ }^{24}$ Variability in ultrasonography technique, interpretation and recommendations for treatment can occur, ${ }^{38}$ as well as technical errors, including failure to correctly identify anatomical landmarks. ${ }^{36}$

The GP may consider medical imaging in patients with an equivocal examination, for selective screening for infants with risk factors or to provide further anatomical information regarding the diagnosis. ${ }^{17}$ Ultrasonography is the modality of choice until four months of age. ${ }^{17}$ Where there is no specialised paediatric ultrasonography service or doubt regarding adequacy, the threshold for referral to a paediatric orthopaedic surgeon in the presence of risk factors or equivocal physical examination findings may be lower. Between four and six months of age, X-ray or ultrasonography can be used; after six months of age, X-ray is preferred. ${ }^{17}$ Increased selective ultrasonography screening for babies with DDH risk factors has been associated with an increase in late-diagnosed DDH in South Australian children without traditional risk factors. ${ }^{4}$ Selective ultrasonography screening may lead overall to a false sense of security ${ }^{9}$ and less vigilance with DDH clinical examination and surveillance in all children. The American Academy of Pediatrics (AAP) recommends routine DDH screening either with ultrasonography at six weeks or X-ray at four months for female breech babies and considers it optional for female babies who have a first-degree relative with DDH and for breech boys. ${ }^{17}$

Recommendations for specialist referral following DDH assessment are presented in Table 1. The Royal Children's Hospital Melbourne provides DDH information that is appropriate for practitioners involved in DDH assessment(available at www.ddheducation.com). ${ }^{39}$

\section{Surveillance versus screening}

Screening usually refers to a short-term, cross-sectional process in a population at risk, whereas surveillance describes

Table 1. Recommendations for referral to a paediatric orthopaedic surgeon following developmental dysplasia of the hip assessment

\begin{tabular}{ll}
\hline Absolute indications & Relative indications \\
\hline Hip is dislocated or dislocatable & Any equivocal examination finding \\
Ultrasound reports $<40 \%$ femoral head & (eg bilateral reduced hip abduction) \\
coverage at six weeks of age or older & Ultrasound reports immaturity at \\
Ultrasound reports signs of hip dysplasia & six weeks and no improvement in \\
X-ray after 4-6 months of age reports & femoral head coverage after repeat \\
signs of hip dysplasia & ultrasonography in further six weeks \\
Risk factors and/or examination findings & Risk factors for developmental \\
meet local guidelines for referral & dysplasia of the hip (particularly breech \\
\end{tabular}

Unilateral reduced hip abduction, especially after eight weeks of age

Doctor or parental concern

Leg length difference on clinical examination

Unilateral toe walking

Table developed with the assistance of members of the Australian Paediatric Orthopaedic Society a long-term process where screening examinations are repeated at intervals of time. ${ }^{9}$ Dislocated hips are diagnosed in Australian children up to five years of age, ${ }^{2-4}$ with the majority of diagnoses above three months of age in walkingage children who self-present. ${ }^{2-4}$ More frequent doctor-initiated assessments can reduce the age at diagnosis. ${ }^{17}$ Peaks in diagnosis occur in conjunction with scheduled hip check visits at three and six months, supporting repeated hip examination outside the newborn period. ${ }^{4,28,29}$ Prescribed ongoing examinations are included in some Australian DDH screening guidelines. ${ }^{10-16}$ The AAP recommends hip examination at well-baby checks at two to four days and by one, two, four, six, nine and 12 months of age. ${ }^{17}$ The principle of multiple assessments until walking age is more important than the exact timing of the examinations.

There are advantages to supplementing DDH screening ${ }^{10-16}$ with regular GP DDH assessments. The GP has an ongoing relationship with the child, can identify changes in examination findings and provide preventive health advice, including avoidance of lower limb swaddling. ${ }^{9}$ The GP can order medical imaging when indicated and refer promptly for treatment, minimising communication breakdowns 
and treatment delays that occur with screening programs generally. ${ }^{9}$ Young children visit the GP for a range of reasons, including immunisations and common childhood illnesses, providing opportunities for repeated DDH assessment.

\section{Discussion}

Early DDH detection and treatment harnesses the maximal growth potential of the infant hip, with increased likelihood of treatment success, fewer invasive procedures and fewer complications. ${ }^{3,4,17}$ Late diagnosis may occur as a result of screening failures, ${ }^{3}$ inappropriate swaddling ${ }^{22,23}$ or progression of pathology; efforts to minimise late cases may be hampered by lack of consensus in screening guidelines. ${ }^{31}$ While future developments in our understanding of the genetic component may lead to targeted investigations of individuals who are at risk, ${ }^{18}$ currently we must search for hip pathology in all children.

\section{Key points}

- Rates of late-diagnosed DDH cases have increased in Australia.

- DDH assessment focuses on clinical examination, supplemented by risk factor identification and consideration of imaging.

- DDH surveillance requires ongoing assessments until walking age.

- GP assessment and surveillance may maximise DDH detection in infancy and lower the age of detection for children who develop signs beyond the newborn period.

\section{Author}

Nicole Williams BMedSc (Hons), BMed, Grad Dip Sports Med, FRACS, FAOrthA, Paediatric Orthopaedic Surgeon, Women's and Children's Hospital, Adelaide, SA; Centre for Orthopaedic and Trauma Research, University of Adelaide, SA. nicole.williams4@sa.gov.au Competing interests: None.

Provenance and peer review: Commissioned, externally peer reviewed.

Declaration: The author is a medical advisor to the International Hip Dysplasia Institute, Board Member of Healthy Hips Australia and Member of the Australian Paediatric Orthopaedic Society.

\section{References}

1. Price CT, Ramo BA. Prevention of hip dysplasia in children and adults. Orthop Clin North Am 2012;43(3):269-79. doi: 10.1016/j.ocl.2012.05.001.

2. Birke $O$, Wallgren $L$, Bridge $C$. Late open reduction and pelvic \pm femoral osteotomy for increasing numbers of missed walking age DDH hip dislocation vs early treatment with Pavlik harness - Evaluation at 5 years follow-up [unpublished presentation]. Noosa: Australian Paediatric Orthopaedic Society Annual Scientific Meeting, 22 Sept 2012.

3. Lisle R, Boekelaar M, Stannage K, Whitewood C Delayed diagnosis of developmental dislocation of the hip: The Western Australian experience. ANZ J Surg 2012;82(9):612-15. doi: 10.1111/j.14452197.2012.06110.x.

4. Studer K, Williams N, Antoniou G, et al. Increase in late diagnosed developmental dysplasia of the hip in South Australia: Risk factors, proposed solutions. Med J Aust 2016;204(6):240.

5. Klisic PJ. Congenital dislocation of the hip A misleading term: Brief report. J Bone Joint Surg (Br) 1989;71(1):136.

6. Shorter D, Hong T, Osborn DA. Screening programmes for developmental dysplasia of the hip in newborn infants. Cochrane Database Syst Rev 2011;(9):CD004595. doi: 10.1002/14651858. CD004595.pub2.

7. Shaw BA, Segal LS. Evaluation and referral for developmental dysplasia of the hip in infants. Pediatrics 2016;138(6).

8. Paton RW. Screening in Developmental Dysplasia of the Hip (DDH). Surgeon 2017;15(5):290-96. doi: 10.1016/j.surge.2017.05.002.

9. Wilson J JG. Public health papers no 34: Principles and practice of screening for disease. Geneva: World Health Organization, 1968.

10. Royal Prince Alfred Hospital. Newborn care guidelines: Developmental dysplasia of the hip. Camperdown, NSW: RPA, 2004. Available at www. slhd.nsw.gov.au/rpa/neonatal/html/docs/DDHips. pdf [Accessed 18 February 2018].

11. NSW Government. Screening, assessment and management of developmental dysplasia of the hip. Clinical practice guideline, resource manual. NSW: NSW Government, 2011. Available at www.nchn.org.au/docs/Man-DDH.pdf [Accessed 18 February 2018].

12. Department of Health. Queensland clinical guidelines: Routine newborn assessment. Canberra: DoH, 2014. Available at www.health.qld. gov.au/_data/assets/pdf_file/0029/141689/gnewexam.pdf [Accessed 18 February 2018].

13. Royal Hospital for Women. Local operating procedures: Developmental dysplasia of the hip screening. NSW: Royal Hospital for Women, 2016. Available at www.seslhd.health.nsw.gov. au/rhw/Newborn_Care/Guidelines/Medical/ nccmedicaldysplasia.pdf [Accessed 18 February 2018].

14. Department for Health and Ageing, Government of South Australia. South Australian perinatal practice guidelines: Neonatal hip screening and management of developmental dysplasia of the hip. SA: SA Health, updated 20 June 2017. Available at www.sahealth.sa.gov.au/wps/wcm/ connect/Public+Content/SA+Health+Internet [Accessed 18 February 2018].

15. Victoria State Government. Developmental dysplasia of the hip in neonates. Vic: Health Vic, date unknown. Available at www2.health.vic.gov. $\mathrm{au} /$ hospitals-and-health-services/patient-care/ perinatal-reproductive/neonatal-ehandbook/ congenital-abnormalities/hip-dysplasia [Accessed 28 February 2018].
16. Government of Western Australia, Department of Health. Clinical practice guideline: Developmental Dysplasia of the Hips (DDH). WA: DoH, updated 27 June 2017. Available at www.kemh.health. wa.gov.au/services/nccu/guidelines/documents/ DDH.pdf [Accessed 28 February 2018].

17. Clinical practice guideline: Early detection of developmental dysplasia of the hip. Committee on Quality Improvement, Subcommittee on Developmental Dysplasia of the Hip. American Academy of Pediatrics. Pediatrics 2000;105(4 Pt 1):896-905.

18. Hatzikotoulas K, Roposch A, Shah K, et al. The genetic epidemiology of developmental dysplasia of the hip: A genome-wide association study harnessing national clinical audit data. bioRxiv 2017 [EPub ahead of print]. doi: https://doi. org/10.1101/154013.

19. Yamamuro T, Ishida K. Recent advances in the prevention, early diagnosis, and treatment of congenital dislocation of the hip in Japan. Clin Orthop Relat Res 1984;(184):34-40.

20. Graham SM, Manara J, Chokotho L, Harrison WJ. Back-carrying infants to prevent developmental hip dysplasia and its sequelae: Is a new public health initiative needed? J Pediatr Orthop 2015;35(1):57-61. doi: 10.1097/ BPO.0000000000000234.

21. International Hip Dysplasia Institute. Website. Orlando: International Hip Dysplasia Institute, 2018. Available at https://hipdysplasia.org [Accessed 5 March 2018].

22. Mulpuri K, Schaeffer EK, Andrade J, et al. What risk factors and characteristics are associated with late-presenting dislocations of the hip in infants? Clin Orthop Relat Res 2016;474(5):1131-37. doi: 10.1007/s11999-015-4668-0.

23. Williams N, Foster BK, Cundy PJ. Is swaddling damaging our babies' hips? Med J Aust 2012;197(5):272.

24. Charlton SL, Schoo A, Walters L. Early dynamic ultrasound for neonatal hip instability: Implications for rural Australia. BMC Pediatr 2017;17(1):82. doi: 10.1186/s12887-017-0830-z.

25. Graf R. Hip sonography: Diagnosis and management of infant hip dysplasia. 2 nd edn. Berlin: Springer, 2006.

26. Upasani VV, Bomar JD, Matheney TH, et al. Evaluation of brace treatment for infant hip dislocation in a prospective cohort: Defining the success rate and variables associated with failure. J Bone Joint Surg Am 2016;98(14):1215-21. doi: 10.2106/JBJS.15.01018.

27. Studer K, Williams N, Studer P, et al. Obstacles to reduction in infantile developmental dysplasia of the hip. J Child Orthop 2017;11(5):358-66. doi: 10.1302/1863-2548.11.170031.

28. Azzopardi T, Van Essen P, Cundy PJ, Tucker G, Chan A. Late diagnosis of developmental dysplasia of the hip: An analysis of risk factors. J Pediatr Orthop B 2011;20(1):1-7. doi: 10.1097/ BPB.0b013e3283415927.

29. Sharpe P, Mulpuri K, Chan A, Cundy PJ. Differences in risk factors between early and late diagnosed developmental dysplasia of the hip. Arch Dis Child Fetal Neonatal Ed 2006;91(3):F158-62.

30. Roposch A, Liu LQ, Hefti F, Clarke NM, Wedge JH. Standardized diagnostic criteria for developmental dysplasia of the hip in early infancy. Clin Orthop Relat Res 2011;469(12):3451-61. doi: 10.1007/ s11999-011-2066-9.

31. Wright $S$, Cotterell E, Schmidt D. Screening for developmental dysplasia of the hip in a rural health district: An analysis of practice. Aust J Rural Health 2017 [EPub ahead of print]. doi: 10.1111/ ajr.12400. 
32. Mubarak SJ. In search of Ortolani: The man and the method. J Pediatr Orthop 2015;35(2):210-16 doi: 10.1097/BPO.0000000000000250.

33. Barlow TG. Early diagnosis and treatment of congenital dislocation of the hip. J Bone Joint Surg (Br) 1962;44(2):292-301.

34. Tschauner C, Fürntrath F, Saba Y, Berghold A, Radl R. Developmental dysplasia of the hip: Impact of sonographic newborn hip screening on the outcome of early treated decentered hip joints - A single center retrospective comparative cohort study based on Graf's method of hip ultrasonography. J Child Orthop 2011;5(6):415-24. doi: 10.1007/s11832-011-0366-y.

35. Thallinger C, Pospischill R, Ganger R, Radler C, Krall C, Grill F. Long-term results of a nationwide general ultrasound screening system for developmental disorders of the hip: The Austrian hip screening program. J Child Orthop 2014;8(1):3-10. doi: 10.1007/s11832-014-0555-6.

36. Graf R, Mohajer M, Plattner F. Hip sonography update. Quality-management, catastrophes Tips and tricks. Med Ultrason 2013;15(4):299-303.

37. Omeroğlu H. Use of ultrasonography in developmental dysplasia of the hip. J Child Orthop 2014;8(2):105-13. doi: 10.1007/s11832-014-0561-8.
38. Orak MM, Onay T, Cağırmaz T, Elibol C, Elibol FD, Centel $\mathrm{T}$. The reliability of ultrasonography in developmental dysplasia of the hip: How reliable is it in different hands? Indian J Orthop 2015;49(6):610-14. doi: 10.4103/0019-5413.168753.

39. Donnan L. Developmental dysplasia of the

hip. Vic: Department of Education and Early Childhood Development, State Government of Victoria, 2011. Available at www.ddheducation.com [Accessed 23 April 2018]. 